\title{
Diagnosis and management of developmental coordination disorder
}

\author{
Susan R. Harris PhD PT, Elizabeth C.R. Mickelson BSc(PT) MD, Jill G. Zwicker PhD OT(C)
}

$\mathrm{D}$ evelopmental coordination disorder is a common neuromotor condition, affecting about $5 \%-6 \%$ of school-aged children; ${ }^{1}$ this equates to more than 400000 Canadian children. ${ }^{2}$ Despite its prevalence, developmental coordination disorder may be underrecognized by health care professionals. ${ }^{1,3}$ In a recent online survey of 1297 parents, teachers and physicians, only $41 \%$ of pediatricians and $23 \%$ of general practitioners had knowledge of the condition; the physician respondents were from Canada, the United States and the United Kingdom. ${ }^{3}$ Furthermore, only $23 \%$ of the pediatricians and $9 \%$ of the general practitioners surveyed had ever diagnosed developmental coordination disorder. The vast majority of the physicians reported the need for more education about the condition. ${ }^{3}$

In this article, we define developmental coordination disorder and describe how it is diagnosed, discuss associated risk factors and the consequences of the condition on physical, social and emotional well-being, and present the evidence that supports promising interventions. This review is supported by the findings of recent systematic reviews as well as evidence-based recommendations from the European Academy of Childhood Disability ${ }^{1}$ (Box 1).

\section{What is developmental coordination disorder?}

As described in the American Psychiatric Association's latest edition of the Diagnostic and Statistical Manual of Mental Disorders (DSM-5), ${ }^{4}$ the

\section{Box 1: Evidence used in this review}

We searched PubMed and Embase (OvidSP) from January 1992 to August 2014 using the terms "developmental coordination disorder" alone and combined with "diagnosis," "intervention" and "risk factors." A total of 871 articles were identified. Much of the evidence cited in the current review comes from eight systematic reviews, one scoping review, and the clinical practice guideline of the European Academy of Childhood Disability, all published from 2011 onward. child with developmental coordination disorder has motor coordination below expectations for his or her chronologic age, may have been described as "clumsy" and may have had delays in early motor milestones, such as walking and crawling. Difficulties with coordination of either gross or fine motor movements, or both, interfere with academic achievement or activities of daily living. Coordination difficulties do not relate to a medical condition or disease (e.g., cerebral palsy, muscular dystrophy, visual impairment or intellectual disability). If intellectual disability is present, the motor difficulties are in excess of those expected for the child's IQ.

In the previous DSM edition (DSM-IV-TR), ${ }^{5}$ developmental coordination disorder was included under the broad category of "learning disorders"; in DSM-5, it is subcategorized as a motor disorder within the broader category of "neurodevelopmental disorders." ${ }^{4}$ An additional criterion included in DSM-5 is that the onset of symptoms occurs during the developmental period.

\section{What are the risk factors?}

A 2011 systematic review of 16 studies involving school-aged children showed significantly greater odds of developmental coordination disorder among children who had very low birth weights $(<1500 \mathrm{~g})$ or were very preterm $(<32 \mathrm{wk})$ than among age-matched controls born at term with normal birth weights (odds ratio [OR] 6.29, 95\% confidence interval [CI] 4.37-9.05] to $8.66,95 \%$ CI 3.40-22.07). ${ }^{6}$ Boys are 1.7 to 2.8 times more likely than girls to have the disorder. ${ }^{7,8}$
Competing interests: None declared.

This article has been peer reviewed.

Correspondence to:

Susan Harris,

susan.harris@ubc.ca

CMAJ 2015. DOI:10.1503 /cmaj.140994

\section{KEY POINTS}

- Developmental coordination disorder is a prevalent childhood disability affecting $5 \%-6 \%$ of school-aged children.

- The disorder has long been underrecognized and seldom diagnosed by general practitioners and pediatricians.

- Many of the motor and psychosocial difficulties associated with the disorder continue into adulthood.

- Recent systematic reviews and practice guidelines have identified successful, evidence-based interventions for children with developmental coordination disorder. 
Additional risk factors were explored in a large $(n=33354)$ national cohort study of Danish children followed up for seven years. ${ }^{7}$ In addition to male sex, other factors associated with developmental coordination disorder included very preterm birth (OR 6.28, 95\% CI 3.99-9.89), small for gestational age (OR 1.74, 95\% CI 1.462.08) and independent walking at 15 months or later (OR 3.05, 95\% CI 2.57-3.60). ${ }^{7}$ A retrospective study involving children 4-5 years of age confirmed the associations with male sex and low birth weight; it also found an association with postnatal exposure to steroids. ${ }^{8}$ Children with developmental coordination disorder are at increased risk of being overweight or obese. ${ }^{9}$

Box 2: Questions to ask parents when a child is referred for possible developmental coordination disorder

- Was your child born preterm? If so, how early?

- How much did your child weigh at birth?

- At what age did your child first walk independently?

- Would you (or anyone else) describe your child as "clumsy"?

- Does your child have difficulty in activities of daily living, such as dressing (including buttoning shirts and tying shoelaces), brushing teeth, and using a knife and fork when eating?

- At what age did your child learn to ride a bicycle (without training wheels)?

- Does your child have difficulty with fine motor activities such as handwriting (cursive), printing, or cutting with scissors? Does s/he switch hand dominance?

- Does your child have difficulty with gross motor activities, such as throwing or kicking a ball, competing in team sports at school or in the community, or participating successfully in physical education classes?

- Has anyone else in your family had a diagnosis of developmental coordination disorder, attention-deficit/hyperactivity disorder or specific learning disabilities?

Box 3: Components of the neuromotor examination relevant for young children at risk of developmental coordination disorder ${ }^{12}$

\section{Cranial nerve examination}

- Observe eye movements

- Check response to visual fields by confrontation

- Observe pupillary responses to light

- Observe eye opening and closing

- Observe facial expression

- Have child drink through a straw or blow kisses

Strength and flexibility

- Palpate muscle bulk and texture

- Assess joint flexibility

- Assess quality and intensity of grasp

Motor planning

- Observe functional gross motor skills (e.g., running, hopping, skipping, balancing on one foot)

- Observe functional fine motor skills (e.g., buttoning, zipping, cutting with scissors, tying shoelaces)

- Determine hand dominance (or lack thereof)
In a prospective, population-based cohort study in southwestern England, probable developmental coordination disorder was identified in 346 children aged 7-9. ${ }^{10}$ Risk factors associated with the disorder were difficulties with attention (OR 1.94, 95\% CI 1.17-3.24), social communication (OR 1.87, 95\% CI 1.15-3.04), repetition of nonwords (e.g., unfamiliar spoken nonwords, such as barrazon, that the child is asked to repeat) (OR 1.83, 95\% CI 1.26-2.66), spelling (OR 2.81, 95\% CI 2.03-3.90) and reading (OR $3.35,95 \%$ CI $2.36-4.77) .{ }^{10}$

\section{When is the disorder suspected and how is it screened?}

Developmental coordination disorder could be present in a child whose parents, caregiver or schoolteacher expresses concerns that he or she is unusually clumsy and is showing persistent delays in gross or fine motor milestones. In our clinical experience, difficulties with oral motor coordination, such as closing lips to blow bubbles or blowing out birthday candles, may also be reported. Box 2 outlines a series of questions a general practitioner can ask the parent or caregiver when a child is referred with these types of concerns. The questions are in part evidence-based (e.g., preterm birth, age at independent walking) and in part based on our clinical experience.

If the parent answers affirmatively to some or all of the screening questions, and if other possible causes of motor difficulties (e.g., cerebral palsy) have been ruled out by careful history taking and physical examination, it is prudent to refer the child for expert assessment.

\section{Screening questionnaires}

In addition to the questions posed in Box 2, the general practitioner should ask the parent to complete the Developmental Coordination Disorder Questionnaire $^{11}$ (either before or after the initial appointment). This 15 -item questionnaire helps to identify children 5-15 years old who have developmental coordination disorder and is the best of those that have been evaluated. ${ }^{1}$ The Developmental Coordination Disorder Questionnaire and scoring criteria are available at no cost at www.dcdq.ca. Based on the child's age and total score, the general practitioner can then determine whether the child may have the disorder or is unlikely to be affected.

\section{History}

As part of the general practitioner's clinical assessment, the family history should include information on developmental coordination disorder, neurologic disorders, mental health concerns 
and the family's social situation. The child's history should include details of the pregnancy, birth, age of attainment of motor milestones, school performance and any previous or current disorders, especially neurologic or sensory concerns. ${ }^{1}$ Details of the child's current gross motor and fine motor difficulties and performance in activities of daily living should also be explored. ${ }^{1}$

If information from the child's teacher is available about the child's academic performance and concerns about the child's motor functioning at school, attention difficulties or specific learning disorders, it should be included in the physician's initial assessment report.

\section{Clinical examination}

Other possible causes of the child's motor difficulties (e.g., cerebral palsy, muscular dystrophy, systemic illness) should be ruled out through a comprehensive clinical examination that includes the neuromotor status, medical status, sensory status and behavioural status. ${ }^{1}$ The child's cognitive status will also need to be assessed if learning difficulties have been reported by the teacher. Body mass index should be part of the general physical exam, because children with developmental coordination disorder have a propensity to be overweight or obese. ${ }^{9}$

The American Academy of Pediatrics has outlined components of the neuromotor examination appropriate for young children. ${ }^{12}$ Most of these are relevant for the examination of young, schoolaged children at risk of developmental coordination disorder and are summarized in Box 3 .

\section{How is the disorder diagnosed?}

According to recommendations from the European Academy of Childhood Disability, developmental coordination disorder should be diagnosed by a multidisciplinary team of professionals qualified to examine the specific DSM-5 criteria for the disorder. ${ }^{1}$ Ideally, the team should include a physician (e.g., child psychiatrist, developmental pediatrician, child neurologist) and an occupational therapist or physical therapist trained in the standardized motor tools used to assess children suspected of having the disorder. Box 4 outlines the diagnostic criteria for developmental coordination disor$\mathrm{der}^{4}$ and includes examples of how these criteria can be assessed. ${ }^{1,11,13-18}$

\section{What conditions may coexist with the disorder?}

Children who have developmental coordination disorder frequently have other childhood disorders (e.g., attention-deficit/hyperactivity disorder [ADHD], autism spectrum disorder or specific learning disabilities). ${ }^{1,6-8}$ Whereas the DSM-IVTR did not allow for concurrent diagnoses, ${ }^{5}$ the DSM-5 does. ${ }^{4}$

Children with developmental coordination disorder (with or without ADHD) have been found to be at increased risk of anxiety and depression. ${ }^{19,20}$ In one study, children who had the disorder without ADHD reported depressive symptoms five times more often, and anxiety symptoms about one and a half times more often, than typical peers. ${ }^{19}$

Other problems commonly associated with developmental coordination disorder are psychosocial difficulties, ${ }^{21}$ overweight or obesity, ${ }^{9,22}$ hypermobility of joints, ${ }^{23}$ compromised physical

Box 4: Diagnostic criteria for developmental coordination disorder

A. The acquisition and execution of coordinated motor skills is substantially below that expected given the individual's chronological age and opportunity for skill learning and use. Difficulties are manifested as clumsiness (e.g., dropping or bumping into objects) as well as slowness and inaccuracy of performance of motor skills (e.g., catching an object, using scissors or cutlery, handwriting, riding a bike or participating in sports).

How to assess this criterion: According to recent review articles, ${ }^{13,14}$ the most widely used motor test to assist in the diagnosis of this disorder is the Movement Assessment Battery for Children, second edition (Movement ABC-2). ${ }^{15}$ Another test often used is the Bruininks-Oseretsky Test of Motor Proficiency, second edition. ${ }^{16}$ Both tests are normreferenced, reliable and valid in diagnosing developmental coordination disorder.

B. The motor skills deficit in criterion A significantly and persistently interferes with activities of daily living appropriate to chronological age (e.g., self-care and self-maintenance) and affects academic/school productivity, prevocational and vocational activities, leisure and play.

How to assess this criterion: Parents should be asked about their child's performance and length of time to learn motor tasks, such as dressing, tying shoelaces, brushing teeth, using a knife and fork, and printing and handwriting. In addition, checklists or questionnaires for parents and teachers can be helpful, such as the Developmental Coordination Disorder Questionnaire (DCDQ) ${ }^{11}$ and the Movement ABC-2 checklist. ${ }^{17}$ The Little DCDQ is currently undergoing validation to identify preschoolaged children with the disorder. ${ }^{18}$

C. Onset of symptoms is in the early developmental period.

How to assess this criterion: The pattern and trajectory of the child's motor development should be ascertained to determine whether motor delay was present in early life. Parents can be questioned about their child's early developmental history and milestones (Box 2). Although some children with developmental coordination disorder may have delayed motor milestones, many do not.

D. The motor skills deficits are not better explained by intellectual disability (intellectual developmental disorder) or visual impairment and are not attributable to a neurologic condition affecting movement (e.g., cerebral palsy, muscular dystrophy, degenerative disorder).

How to assess this criterion: To rule out intellectual disability, a standardized IQ test administered by a school psychologist is useful; however, a formal IQ assessment may not be necessary if there has been typical achievement at school. ${ }^{1}$ As with screening, a neurologic examination is important to rule out other medical conditions that could explain impaired motor development. ${ }^{1}$ A family physician or optometrist could rule out impairments in visual acuity. ${ }^{1}$ 
fitness, ${ }^{24-26}$ and decreased participation in dailyliving, physical and social activities. ${ }^{27,28}$

In a recent systematic review on the influence of developmental coordination disorder on physical, psychological and social functioning, the authors concluded that "children with DCD report lower self-efficacy and competence in physical and social domains, experience greater symptoms of depression and anxiety, and display more externalizing behaviours when compared to typically developing children." ${ }^{21}$

\section{How is the disorder managed?}

Children with developmental coordination disorder typically receive different types of therapies (e.g., occupational therapy or physical therapy) aimed at improving their motor performance. For children who also have ADHD, drug therapies (e.g., methylphenidate) have been used. Recent evidence is summarized below.

In a well-executed systematic review and metaanalysis, the efficacy of various interventions on motor performance in children with developmental

Box 5: Summary of risk factors, concurrent diagnoses, secondary consequences and therapies relevant to developmental coordination disorder

\section{Risk factors}

- Male sex (odds ratio [OR] 1.7-2.8)

- Very preterm birth (<32 wk) or low birth weight $(<1500 \mathrm{~g})$ or both (OR 6.29-8.66) ${ }^{6}$

- Small-for-gestational age (OR 1.74) ${ }^{7}$

- Independent walking at age 15 months or later (OR 3.05) ${ }^{7}$

- Difficulties in attention (OR 1.94), social communication (OR 1.87), non-word repetition (OR 1.83), spelling (OR 2.81) and reading (OR 3.35) $)^{10}$

\section{Concurrent diagnoses}

- Anxiety (16.7\%-33.8\% $)^{19}$ and depression $(9.1 \%-11.8 \%)^{19}$

- Attention-deficit/hyperactivity disorder $(50 \%)^{1}$

- Autism spectrum disorder $(4.1 \%-8.2 \%)^{1}$

- Specific learning disabilities $(17.8 \%-27.5 \%)^{33}$

- Specific language impairment (up to $70 \%)^{1}$

Secondary consequences

- Compromised physical fitness (OR unknown)

- Decreased participation in daily-living, physical and social activities (OR unknown)

- Hypermobility of joints (OR 1.92$)^{23}$

- Mental health difficulties (OR 2.08-4.23) 20

- Overweight or obesity (OR 1.79-2.28)22

Therapies

- Occupational therapy ${ }^{29}$

- Physical therapy ${ }^{29}$

- Task-oriented interventions ${ }^{29}$

- Methylphenidate 29,30

- Dietary supplementation with fatty acids ${ }^{32}$ coordination disorder was examined. ${ }^{29}$ Twenty-six studies (systematic reviews, randomized controlled trials and crossover studies) published between 1995 and 2011 were included and divided into four categories: task-oriented intervention; traditional occupational therapy or physical therapy; process-oriented therapies; and chemical supplements. The task-oriented interventions were aimed at learning specific motor skills that are particularly difficult for the child, and the processoriented therapies focused on more global functions (e.g., sensory integration, visual-motor perception and muscle strength). ${ }^{29}$ The largest effect sizes were shown for task-oriented interventions (weighted Cohen $d\left[d_{w}\right]=0.89$ ) and for occupational or physical therapy $\left(d_{w}=0.83\right)$, with only a weak effect for process-oriented approaches $\left(d_{w}=0.12\right) .^{29}$ Thus, referral to an occupational therapist or physical therapist, or both, who can focus treatment on helping the child perform everyday tasks is recommended.

In this same systematic review, ${ }^{29}$ the effects of methylphenidate on fine motor performance were reported from three studies involving children who had developmental coordination disorder and ADHD; the effect size was medium $\left(d_{w}=0.79\right)$. In a blinded study published after the systematic review, motor performance measured on the Movement Assessment Battery for Children, second edition, was substantially improved when children with developmental coordination disorder and concurrent ADHD were taking methylphenidate. ${ }^{30}$ Unfortunately, we found no studies that evaluated the effects of chemical supplements in children who had developmental coordination disorder without ADHD.

In a 2014 review of prospective clinical trials examining the safety of medications for children with ADHD, long-term ( $\geq 1 \mathrm{yr}$ ) adverse effects associated with methylphenidate included headache, decreased appetite, insomnia, abdominal pain, tics and weight loss (with incidence ranging from about $5 \%$ to $20 \%$ ). ${ }^{31}$ As with other research on this topic, the authors reported that most of the adverse effects were mild or moderate. We found no comparable studies of adverse effects of medications among children with developmental coordination disorder and concurrent ADHD.

In the systematic review mentioned earlier, ${ }^{29}$ only one study, a randomized controlled trial, examined the effects of dietary supplementation with fatty acids. Although there were no changes in motor performance, improvements in reading, spelling and behaviour were reported. ${ }^{32}$

Box 5 summarizes risk factors, concurrent diagnoses, secondary consequences and therapies relevant to developmental coordination disorder. ${ }^{1,6-8,10,19,20,22,23,29,30,32,33}$ 


\section{What is best practice in meeting the needs of children with developmental coordination disorder?}

In a scoping review of more than 500 research studies, practice guidelines and grey literature, Camden and associates ${ }^{34}$ identified 31 documents that focused on children 16 years or younger who had developmental coordination disorder and provided "population-level recommendations." Their qualitative analysis of the information, which included the European Academy of Childhood Disability guideline ${ }^{1}$ and studies from Canada, the United Kingdom, Germany and New Zealand, yielded two overarching themes and five principles for best practice, each supported by multiple references. Not surprisingly, the authors identified the need to increase awareness of the disorder and to coordinate services. The importance of consumer involvement in identifying treatment goals was another important principle they identified, as was the need for evidence-based intervention.

We hope that by sharing these principles and providing relevant resources for parents, physicians and educators (see Resources box at end of article), readers of this review will embrace best practice strategies for developmental coordination disorder. We also apply the results of our review to two fictional cases (Box 6) to assist clinicians in this goal.

\section{What are the lifetime consequences?}

Like children with other types of developmental disabilities, children with developmental coordination disorder do not "outgrow" their disorder. There is far less research on issues in adulthood, however. Several studies have assessed psychosocial, motor and executive functions in adults with developmental coordination disorder. Small

Box 6: Applying the results of this review in clinical practice (fictional cases)

\section{Case 1}

Seven-year-old Daniel is a fraternal twin who was born at 32 weeks' gestation. As twin $B$, he weighed less than his twin sister at birth (1470 g v. $1790 \mathrm{~g}$ ). Daniel's parents report that he was a "late" walker, taking his first steps at 16 months' corrected age, 3 months later than his twin. Daniel's parents and his teacher are concerned that he is having difficulty holding a pencil and learning to print. Although his sister is now able to ride a bicycle independently, Daniel still relies on training wheels. He is not yet able to fully dress himself, having trouble with buttons and tying shoelaces. His parents are worried that he might have a mild form of cerebral palsy; they had been warned about this possibility when he was in the neonatal intensive care unit.

Daniel's parents take him to their family physician and share their concerns and his developmental history with her. His teacher has also provided a written report detailing her concerns about his difficulties with learning to print and asking whether he might have a specific learning disability. Daniel has recently had an IQ test at school, and his intellectual ability is within normal limits.

The family physician notes that Daniel is short for his corrected age and is overweight. She asks him what sports he likes to do, and he replies "None, because I'm not good at any of them and the other kids tease me when I try to play along." On physical examination, the physician notes that Daniel has somewhat low muscle tone, hyperflexible joints and flat feet. She assesses him on the Snellen eye chart and notes that his visual acuity is $20 / 20$.

The physician advises the parents that she does not think Daniel has cerebral palsy but suspects he has developmental coordination disorder and would like to refer him to a pediatrician for further assessment. She also suggests that if the school district has an occupational therapist or physical therapist, one or both of them could assess Daniel's coordination and motor skills. In preparation for these upcoming assessments, the physician gives the parents a copy of the Movement ABC-2 parent/teacher checklist and suggests they complete it before their upcoming appointment with the pediatrician.

\section{Case 2}

Jacob is a nine-year-old boy with an unremarkable birth history and normal developmental milestones. His mother takes him to their family physician because of concerns about how he is managing at home and school. She reports that, although he has always been a little clumsy, Jacob is now being teased and bullied at school because of his poor motor skills. He often gets excluded at recess because he can't run as fast as other children, and no one passes the ball to him. Although Jacob generally does well at school, his teacher reports that his written work is below expectations, with his journal entries and creative writing being much shorter in length and messier than his classmates. His mother continues to cut his food for him because he has difficulty using a knife and fork. Jacob has only recently learned to tie his shoes (a skill most of his peers learned four years earlier). He no longer is interested in riding his bicycle because he is embarrassed that he still needs training wheels, and he shows little interest in physical activities, preferring instead to watch television and play video games. He is starting to make self-deprecating comments, one of his mother's main concerns.

Jacob's medical examination is unremarkable. Given his history of his motor skills interfering with his activities of daily living and school performance, coupled with his challenges in learning new motor skills, the physician asks Jacob's mother to complete the Developmental Coordination Disorder Questionnaire. The results reveal that developmental coordination disorder should be suspected. The physician refers Jacob to occupational therapy for a standardized assessment of his motor skills to confirm that criterion A of the DSM-5 diagnostic criteria (Box 4) is met.

Note: DSM-5 = Diagnostic and Statistical Manual of Mental Disorders, fifth edition; Movement ABC-2 = Movement Assessment Battery for Children, second edition. 
observational studies noted that adults with the disorder had significantly high levels of depressive symptoms and anxiety, ${ }^{35,36}$ as well as decreased levels of participation in everyday life, and lower quality of life and life satisfaction. ${ }^{37}$ In addition, small studies based on self-reported data found that motor skills (e.g., playing team sports, parking a car and handwriting) and executive functioning (e.g., planning ahead and managing money) continued to be compromised in adulthood. ${ }^{38,39}$

A large case-control study of the functional profile of young adults in Israel found that those with probable developmental coordination disorder (based on Adolescents and Adults Coordination Questionnaire scores) performed significantly worse $(p<0.001)$ than controls on measures of academic (e.g., handwriting) and nonacademic (e.g., activities of daily living requiring organization and planning) functioning. ${ }^{40}$

\section{Unanswered questions}

Because developmental coordination disorder affects 5\%-6\% of school-aged children, primary care practitioners will likely have such children in their practice and should be aware of how to screen for this disorder and when and to whom to make a referral. Although research in this area is growing at a remarkable pace, there continues

\section{Resources for parents, physicians and educators}

- Developmental Coordination Disorder Research Clinic, Sunny Hill Health Centre for Children, Vancouver (www.childdevelopment.ca/bc/ ViewDetails. aspx?id=118)

- eMentalHealth.ca: lists of organizations and services for developmental coordination disorder in British Columbia (www.ementalhealth.ca/index. php? $m=$ heading\&ID=34) and Ontario (www.ementalhealth.ca/index. php? $m=$ heading \&ID=34)

- Developmental Coordination Disorder Questionnaire: the most recent version of this brief questionnaire for parents of children aged 5-15 years is available for free at www.dcdq.ca

- CanChild Centre for Childhood Disability Research, McMaster University

- Educational materials and online workshops on causes, assessment, diagnosis and management (http://dcd.canchild.ca/en/)

- Educational materials for physicians (http://dcd.canchild.ca/en/ EducationalMaterials/physicians.asp)

- Review articles and textbook

- Vaivre-Douret L. Developmental coordination disorders: state of the art. Neurophysiol Clin 2014;44:13-23

- Zwicker JG, Missiuna C, Harris SR, et al. Developmental coordination disorder: a review and update. Eur J Paediatr Neurol 2012;16: 573-81

- Cairney J, editor. Developmental coordination disorder and its consequences. Toronto: University of Toronto Press; 2015

- Best practice for medical treatment of attention-deficit/hyperactivity disorder

- Cortese S, Holtman M, Banaschewski T, et al. Practitioner review: current best practice in the management of adverse events during treatment with ADHD medications in children and adolescents. $J$ Child Psychol Psychiatr 2014;54:227-46 to be a pressing need for additional research in the following key areas.

How reliable and valid is screening for developmental coordination disorder in primary care for the eventual diagnosis of the disorder by a multidisciplinary team?

What are the benefits and harms of early intervention for children with the disorder?

Which interventions result in the best outcomes for children and adults with the disorder, especially with respect to participation in dailyliving, physical and social activities?

Does early intervention for children with the disorder result in improved outcomes in adulthood?

\section{References}

1. Blank R, Smits-Engelsman B, Polatajko H, et al.; European Academy for Childhood Disability. European Academy for Childhood Disability (EACD): recommendations on the definition, diagnosis and intervention of developmental coordination disorder (long version). Dev Med Child Neurol 2012;54:54-93.

2. Table 051-0001 Estimates of population, by age group and sex for July 1, Canada, provinces and territories. Ottawa: Statistics Canada; 2013. Available: http://www5.statcan.gc.ca/cansim/ pick-choisir?lang=eng\&p2=33\&id=0510001 (accessed 2014 July 31).

3. Wilson BN, Neil K, Kamps PH, et al. Awareness and knowledge of developmental co-ordination disorder among physicians, teachers and parents. Child Care Health Dev 2013;39:296-300.

4. American Psychiatric Association. Diagnostic and statistical manual of mental disorders. 5th ed. Arlington (VA): American Psychiatric Publishing; 2013.

5. American Psychiatric Association. DSM-IV TR diagnostic and statistical manual of mental disorders. Washington (DC): American Psychiatric Publishing; 2000.

6. Edwards J, Berube M, Erlandson K, et al. Developmental coordination disorder in school-aged children born very preterm and/ or at very low birth weight: a systematic review. J Dev Behav Pediatr 2011;32:678-87.

7. Faebo Larsen R, Hvas Mortensen L, Martinusson T, et al. Determinants of developmental coordination disorder in 7-year-old children: a study of children in the Danish National Birth Cohort. Dev Med Child Neurol 2013;55:1016-22.

8. Zwicker JG, Yoon SW, MacKay M, et al. Perinatal and neonatal predictors of developmental coordination disorder in very low birth weight children. Arch Dis Child 2013;98:118-22.

9. Hendrix CG, Prins MR, Dekkers H. Developmental coordination disorder and overweight and obesity in children: a systematic review. Obes Rev 2014;15:408-23.

10. Lingam R, Golding J, Jongmans MJ, et al. The association between developmental coordination disorder and other developmental traits. Pediatrics 2010;126:e1109-18.

11. Wilson BN, Crawford SG, Green D, et al. Psychometric properties of the revised Developmental Coordination Disorder Questionnaire. Phys Occup Ther Pediatr 2009;29:182-202.

12. Noritz GH, Murphy NA. Motor delays: early identification and evaluation. Pediatrics 2013;131:e2016-27.

13. Martin KS, Westcott S, Wrotniak BH. Diagnosis dialogue for pediatric physical therapists: hypotonia, developmental coordination disorder, and pediatric obesity as examples. Pediatr Phys Ther 2013;25:431-43.

14. Kirby A, Sugden D, Purcell C. Diagnosing developmental coordination disorders. Arch Dis Child 2014;99:292-6.

15. Henderson SE, Sugden DA. Movement Assessment Battery for Children - second edition. London (UK): Psychological Corporation; 2007.

16. Bruininks RH, Bruininks BD. Bruininks-Oseretsky test of motor proficiency, second edition. Minneapolis: Pearson Assessments; 2005

17. Henderson SE, Sugden DA. Movement Assessment Battery for Children - second edition: examiner's manual. London (UK): Harcourt Assessment; 2007.

18. Wilson BN, Creighton D, Crawford SG, et al. Psychometric properties of the Canadian Little Developmental Questionnaire for Preschool Children. Phys Occup Ther Pediatr 2014 Dec. 2 [Epub ahead of print]. 
19. Missiuna C, Cairney J, Pollock N, et al. Psychological distress in children with developmental coordination disorder and attention-deficit hyperactivity disorder. Res Dev Disabil 2014;35:1198-207.

20. Lingam R, Jongmans MJ, Ellis M, et al. Mental health difficulties in children with developmental coordination disorder. Pediatrics 2012;129:e882-91.

21. Zwicker JG, Harris SR, Klassen AF. Quality of life domains affected in children with developmental coordination disorder: a systematic review. Child Care Health Dev 2013;39:562-80.

22. Zhu YC, Cairney J, Li YC, et al. High risk for obesity in children with a subtype of developmental coordination disorder. Res Dev Disabil 2014;35:1727-33.

23. Jelsma LD, Geuze RH, Klerks MH, et al. The relationship between joint mobility and motor performance in children with and without the diagnosis of developmental coordination disorder. BMC Pediatr 2013;13:35.

24. Rivilis I, Hay J, Cairney J, et al. Physical activity and fitness in children with developmental coordination disorder: a systematic review. Res Dev Disabil 2011;32:894-910.

25. van der Hoek FD, Stuive I, Reinders-Messelink HA, et al. Healthrelated physical fitness in Dutch children with developmental coordination disorder. J Dev Behav Pediatr 2012;33:649-55.

26. Oudenampsen C, Holty L, Stuive I, et al. Relationship between participation in leisure time physical activities and aerobic fitness in children with DCD. Pediatr Phys Ther 2013;25:422-9.

27. Magalhães LC, Cardoso AA, Missiuna C. Activities and participation in children with developmental coordination disorder: a systematic review. Res Dev Disabil 2011;32:1309-16.

28. Sylvestre A, Nadeau L, Charron L, et al. Social participation by children with developmental disorder compared to peers. Disabil Rehabil 2013;35:1814-20

29. Smits-Engelsman BC, Blank R, van der Kaay AC, et al. Efficacy of interventions to improve motor performance in children with developmental coordination disorder: a combined systematic review and metaanalysis. Dev Med Child Neurol 2013;55:229-37.

30. Bart O, Daniel L, Dan O, et al. Influence of methylphenidate on motor performance and attention in children with developmental coordination disorder and attention deficit hyperactivity disorder. Res Dev Disabil 2013;34:1922-7

31. Clavenna A, Bonati M. Safety of medicines used for ADHD in children: a review of published prospective clinical trials. Arch Dis Child 2014;99:866-72.

32. Richardson AJ, Montgomery P. The Oxford-Durham study: a randomized, controlled trial of dietary supplementation with fatty acids in children with developmental coordination disorder. Pediatrics 2005;115:1360-6

33. Margari L, Buttiglione M, Craig F, et al. Neuropsychological comorbidities in learning disorders. BMC Neurol 2013;13:198.

34. Camden C, Wilson B, Kirby A, et al. Best practice principles for management of children with developmental coordination disorder (DCD): results of a scoping review. Child Care Health Dev 2015;41:147-59.

35. Kirby A, Williams N, Thomas M, et al. Self-reported mood, general health, wellbeing and employment status in adults with suspected DCD. Res Dev Disabil 2013;34:1357-64.

36. Hill EL, Brown D. Mood impairments in adults previously diagnosed with developmental coordination disorder. J Ment Health 2013; 22:334-40

37. Tal-Saban M, Ornoy A, Parush S. Young adults with developmental coordination disorder: a longitudinal study. Am J Occup Ther 2014; 68:307-16.

38. Kirby A, Edwards L, Sugden D. Emerging adulthood in developmental co-ordination disorder: parent and young adult perspectives. Res Dev Disabil 2011;32:1351-60.

39. de Oliveira RF, Wann JP. Driving skills in young adults with developmental coordination disorder: Maintaining control and avoiding hazards. Hum Mov Sci 2012;31:721-9.

40. Tal-Saban M, Zarka S, Grotto I, et al. The functional profile of young adults with suspected developmental coordination disorder (DCD). Res Dev Disabil 2012;33:2193-202.

Affiliations: Departments of Physical Therapy (Harris), Pediatrics (Harris, Mickelson, Zwicker), and Occupational Science and Occupational Therapy (Zwicker), Faculty of Medicine, University of British Columbia, Vancouver, BC

Contributors: All of the authors contributed substantially to the conception and design of the article, drafted the manuscript or revised it critically for important intellectual content, approved the final version to be published and have agreed to be guarantors of the work.

Acknowledgement: Jill Zwicker is funded by the Michael Smith Foundation for Health Research and the Canadian Child Health Clinician Scientist Program. 